\title{
EL TRABAJO DE LA MUJER EN FORTALEZA
}

\author{
FELÍCIA REICHER MADEIRA \\ Centro Brasileiro de Análise e Planejamento
}

\section{INTRODUCCIÓN}

EL PRESENTE TRABAJO forma parte de una tesis en proceso de elaboración. Este documento se trae a esta reunión para ser discutido; no es por lo tanto el resumen de los resultados, ni constituye tampoco un capítulo de la tesis. Lo que nos propusimos hacer era aprovechar la oportunidad de someter a una discusión amplia un intento de síntesis de la línea teórico-metodológica que lo orienta, resaltando los aspectos que consideramos incrementan las discusiones corrientes sobre el tema.

El documento está dividido en tres grandes capítulos además de la introducción. Tanto los capítulos como sus partes no están desarrollados de la misma manera. En parte, esta desigualdad es fruto de la etapa en que se encuentra el trabajo, pero también responde a otras intenciones. Antes de todo se procuró desarrollar las contribuciones que serían por así decirlo "teóricamente más discutibles" y la metodología que de ellas se deriva. Nos referimos de manera específica a la hipótesis de que la eliminación de la producción doméstica del cálculo de la producción de bienes y servicios es ante todo ideológica y que pretende ocultar, a nivel consciente de los agentes sociales, aspectos esenciales de la explotación capitalista.

De manera intencional renunciamos a extendernos en hipótesis y conclusiones que son fundamentales para este capítulo y en el conjunto del trabajo, por estar desarrolladas en los trabajos de Paul Singer -Emprego, produgáo e reprodugáo da FT (1975); A economía urbana de um ponto de vista estrutural: o caso de Salvador (1977), y Elizabeth Jelin, O trabalho feminino na Bahía (1976) y Formas de organizjOQáo da actividade económica e estrutura ocupacional (1974).

El lector que conoce estos textos encontrará repeticiones que no son señaladas a cada paso con la cita correspondiente. En otros puntos encontrará reinterpretaciones de algún aspecto, o nuevas conclusiones de hipótesis desarrolladas en aquellos textos sin que quedara muy clara una contribución específica. En nuestro caso juzgamos innecesario insistir y repetir 
en las referencias bibliográficas por ser conocidas la proximidad física, de fuentes, y de líneas de interpretación con los referidos autores.

El tema relativo a la problemática del empleo en América Latina también se encuentra desarrollado de manera desproporcionada. Este tema fue incluido en el texto en la forma como se presente el documento para esta reunión especialmente. Como por lo general no se ha abordado la problemática del trabajo de la mujer desde esta perspectiva, y el conocimiento de sus principales líneas de interpretación y conclusiones son fundamentales para entender los aspectos en que se aclara y se incrementa con esta nueva propuesta de análisis, se consideró fundamental incluirla.

\section{LA PROBLEMÁTICA DEL EMPLEO}

Una revisión de la bibliografía relativa al tema de la fuerza de trabajo está muy lejos de ser conocida en toda su extensión. Un^ ${ }^{\wedge}$ peculiaridad como la que se evidencia en cada área considerada indica que una gama de factores económicos, demográficos, sociales, psicológicos, ideológicos, etc. actúan de manera ínter/dependiente, pero de las causas de la dinámica| se conoce muy poco.

Las maneras de abordar el problema en los países desarrollados varían considerablemente, por ejemplo desde aquellos estudios que tratan del significado del trabajo diario del individuo hasta la aplicación de teorías de dirección dentro de la familia para establecer estrategias de participación en el mercado de trabajo.

En cuanto a la problemática de la fuerza de trabajo, de manera específica en los países de industrialización dependiente, la gran preocupación de los autores se concentra en algunos aspectos considerados de manera unánime como fundamentales, pero que no por eso dejan de ser menos oscuros. La verdad es que si se utiliza como indicador la literatura existente,, se deduce que el cuadro es extremadamente confuso.

Las posiciones básicas respecto del empleo surgieron a partir de algunas tendencias recientes en América Latina como resultado del estilo de desarrollo adoptado. Las más relevantes se resumen a continuación.'

La urbanización y la modernización del modo de vida fueron, en comparación con los países de industrialización antigua, bastante rápidas y en extremo dependientes de presiones y estímulos no sólo en el plano económico y político, sino también cultural. Una minoría, aunque creciente, de las poblaciones latinoamericanas comenzaron a participar en ocupaciones más productivas y servicios "modernos" y adoptaron también normas de consumo moderno. Estas normas fueron impuestas en cierta forma a la población restante, a través de la penetración de la comunicación masiva y las aspiraciones de consumo, con lo que en cierta forma se debilita cada

\footnotetext{
' Estas conclusiones fueron tomadas de CEPAL, Desarrollo humano, cambio social y crecimiento en América Latina, Santiago de Chile, 1975.
} 
vez más la viabilidad de las formas tradicionales de vida y se genera la necesidad de otras nuevas. Se dio entonces una creciente valorización de lo "moderno", y lo "tradicional" pasó a ser principalmente una forma académica en relación al "pobre", la "baja productividad" y el "marginal".

Los indicadores estadísticos, extraídos de los censos de 1960 y 1970 revelaron todas las inconsistencias y aportaron evidencias de que el estilo del desarrollo era capaz de ofrecer algunas formas de participación más fácilmente que otras.

En términos globales, la escolaridad tiende a crecer mucho, de manera notable en el decenio de los sesenta en cuyo lapso los niveles medio y alto crecieron más rápidamente que el primario. En la mayoría de los países la educación primaria no recibe todavía atención completa y la educación elemental que es ofrecida en las áreas rurales es muy reducida y de mala calidad.

La proporción de la población económicamente activa en ocupaciones urbanas que podrían ser clasificadas como de niveles "medios" y "elevados", también crecieron de manera considerable, pero no en la misma proporción que los niveles educacionales medios y altos. Dado que la educación se expandió de manera más rápida que las ocupaciones correspondientes, los requerimientos de educación formal para la admisión en estas ocupaciones aumentaron continuamente. Todavía en el decenio de los cincuenta, varios estudios educacionales señalaban que los individuios que desempeñaba ocupaciones "medias" y "altas" tendían a estar subeducados para esa posición. A partir de mediados del decenio de los sesenta tendían a estar claramente sobreeducados.

Sin embargo, los datos sugieren que el mayor grado de concentración de los beneficios proporcionados por el desarrollo dependiente no es educacional ni ocupacional, más bien está en la distribución del ingreso. Una comparación entre los datos de 1960 y 1970 muestra que la brecha entre los grupos prJóximos a la cúspide de la pirámide y los grupos próximos a la base, se ensanchó. Al mismo tiempo, parece haber sido una tendencia general el aumento del tamaño relativo de los grupos en la cúspide de la pirámide y próximos a ella, exactamente aquellos que disfrutaban de ingresos en extremo desproporcionados.

El propio significado de las ganancias monetarias se vio afectado y alterado para los portadores del ingreso en todos los niveles. En las bandas de ingreso medio aumentaron bastante las aspiraciones de consumo, con toda probabilidad de manera más rápida que el crecimiento de ingreso, en esencia debido a la repercusión de algunos productos como automóviles, televisores cromáticos, etc. En el estrato de ingreso medio-bajo y tal vez hasta en los estratos más bajos, las aspiraciones de consumo de bienes modernos y los gastos derivados de las necesidades de las complicaciones de la vida urbana ejercieron presiones que acabaron de inducir un reescalonamiento de las prioridades básicas, alterando inclusive la noción de una dieta adecuada. 
Conviene señalar también, como última tendencia para las posiciones más frecuentes en la problemática del empleo, la creciente disminución de las tasas de mortalidad, lo cual ocasionó un aumento en los índices de crecimiento de la población.

La excepcional desigualdad en la distribución del ingreso, el crecimiento de la riqueza al lado de la persistencia de la pobreza masiva, han sido el argumento utilizado con mayor frecuencia para apuntar la inviabilidad del estilo dependiente del desarrollo para incorporar una parte importante de la fuerza de trabajo ( $\mathbf{F} \mathbf{T}$ ), que aumenta con rapidez, en ocupaciones productivas. La atención se centralizó en las reducidas tasas de crecimiento del empleo en la industria moderna y, en contrapartida, en el crecimiento desproporcionado de los sectores de servicios improductivos.

La combinación de estas tendencias para explicar la incapacidad de absorción de mano de obra fue bien sintetizada por Faria. ${ }^{2}$ Así, por un lado se afirma que el capitalismo se desarrolla en América Latina de manera dependiente respecto de los países ya desarrollados, y por otro, que la estructura industrial se edifica en respuesta a pautas de demanda basadas en una distribución del ingreso en extremo desigual, y que por lo tanto el sector industrial tiende a producir bienes propios de una economía de elevado desarrollo, pasando también a depender de la tecnología y los capitales importados, lo cual acarrea la utilización de una constelación de factores que tiende a ser extensiva en el uso de capital. En la medida en que este sistema industrial se desarrolla y altera, libera mano de obra sin ser capaz, en forma simultánea, de absorberla. Factores de orden demográfico, como la disminución de la tasa de mortalidad general e infantil, y el mantenimiento de elevadas tasas de natalidad, tienden a agravar el problema. La subutilización de la población adopta las más variadas facetas - desempleo abierto, subempleo, desempleo disfrazado, desempleo oculto, etc.

En cuanto a las tendencias previstas para estas áreas, a grosso modo se distinguen dos. Están los que defienden la posición de que en los últimos años esta tendencia se ha acentuado, aumentando de manera creciente la subutilización de la mano de obra disponible. ${ }^{3} \mathrm{Y}$ surge una nueva proyección para la economía de estos países a partir del fenómeno de la sobreeducación. ${ }^{4}$ Los adeptos de este nuevo punto de vista sostienen que la tasa de crecimiento global por sí sola sería capaz de incorporar de manera creciente a la población marginada. El argumento central es que el sector moderno de la economía ejerce una gran demanda de mano de obra calificada, y que prácticamente la solución para los problemas del empleo radicaba en unir la educación al crecimiento económico.

Recurrir a los datos empíricos no ha servido mucho para esclarecer el problema. La evidencia empírica al parecer no confirma la creciente

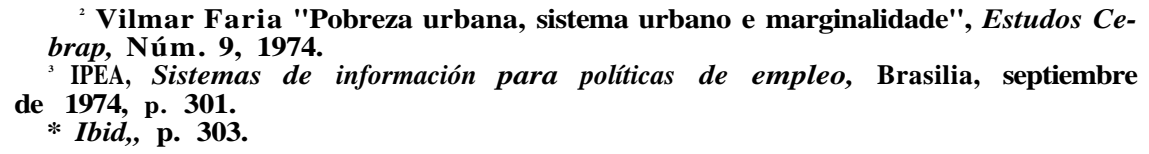


importancia relativa de la subutilización o la marginalización, aunque hasta ahora tampoco ha sido suficiente para demostrar que se puede llegar a una reducción significativa de esa subutilización. Asimismo, si se deja de lado toda la discusión en torno a la problemática de la medida más adecuada de desempleo en los países "subdesarrollados", las tendencias incuestionables observadas han servido como argumento para ambas tesis, de manera principal las evidencias en cuanto a la continua y creciente concentración del ingreso para los primeros y en cuanto a los aumentos en la absorción de mano de obra por el sector secundario para los segundos.

En cuanto a las terapias para atenuar el problema, de manera evidente, en el segundo caso ésta se concreta en el tiempo, no obstante, como recuerda Salm "sin compromisos con fechas, ya que se desconoce quien hubiese aventurado cualquier supuesto sobre el plazo". Para los adeptos de la marginalización creciente, dado que toda la problemática se resume en un desequilibrio entre oferta y demanda de FT, la solución es proponer reformas en uno de los lados de la balanza. La consecuencia natural por el lado de la oferta es proponer políticas de planificación familiar y políticas migratorias, en un intento de limitar el crecimiento de la población; en cuanto a la demanda, las sugerencias son en el sentido de dar preferencia a tecnologías de baja densidad de capital para estimular la demanda de fuerza de trabajo.

La crítica a fondo que se hace a los que proponen estas reformas es que no sólo no se cuestionan la viabilidad de estas propuestas en el capitalismo dependiente, sino que mantienen aún una actitud ingenua sobre sus consecuencias para los sectores que se pretende beneficiar. Es de aquí de donde parte Singer para demostrar que tanto la demanda como la oferta de FT son determinadas por el movimiento de capital y que fundamentalmente la problemática del empleo se deriva del anacronismo entre estos dos procesos. Para el autor, recurrir a técnicas de baja productividad sólo puede "llevar al peor de los dos mundos: la pobreza y el estancamiento".

Para propósitos inmediatos, no interesa profundizar ni avanzar en esta discusión que será retomada de nuevo, en contextos diferentes, a lo largo del trabajo. Por ahora, lo que nos proponemos es mostrar cómo ha sido abordado el trabajo femenino en esta situación de subutilización de mano de obra,, al parecer crónica, cuya explicación y solución están lejos de un consenso.

\section{La participación de la mujer}

La participación de la mujer en las diferentes dimensiones sociales y económicas propiciadas por los cambios descritos en el inciso anterior, difiere de la del hombre, de manera desventajosa para aquélla en la mayoría de los casos.'

s Ibid., p. 303.

Paulo Singer, "Emprego, producao e reproducao da forza de trabalho, CEBRAP, 1976 (Mimeo.).

ONU, Participation of Wornen in Development in Latín America, 1975, México. 
El área en que las ventajas son menos acentuadas es en la educación. Para los niveles primario y medio, el porciento de ganancia así como los índices por sexo han sido particularmente los mismos en la mayoría de los países latinoamericanos. En estos niveles, la escolaridad está mucho más relacionada a la clase social, al nivel de ingreso o a la residencia rural o urbana. Algunas áreas acusan incluso una cierta ventaja educacional para las mujeres en las familias de ingreso más bajo. La hipótesis más común es que este hecho tiene que ver con la menor posibilidad de inserción de la mujer en la fuerza de trabajo. La mujer registra, proporcionalmente, ventajas bastante mayores que los hombres en el crecimiento explosivo de los grados de escolaridad más elevados, a pesar de lo cual y además de las ventajas, en estos niveles ellas se mantienen aún en número bastante menor que los hombres. También es bastante significativo, y por eso merece destacarse, el hecho de que estén representadas de manera desproporcionada en las áreas de humanidades y educación, en las que constituyen la gran mayoría, mientras que su representatividad en otras como ciencias físicas, ingeniería,, agronomía, etc., es bastante reducida.

Algunas elaboraciones hechas con los Censos de 1960 y 1970 muestran que la reducida participación de la mujer en la fuerza de trabajo contrasta, de manera bastante más acentuada que la de los hombres, en cuanto a sus índices de escolaridad.

La participación de la mujer en el crecimiento diferenciado de las ocupaciones "medias" y "altas", mencionadas en el párrafo anterior, fue prácticamente marginal. Las ventajas del empleo femenino se concentran de manera principal en las categorías que compone el sector de servicios productivos. La participación de la mujer en el trabajo industrial tiende a disminuir mientras que en el sector de servicios individuales y comercio ambulante ha permanecido prácticamente constante.

La explicación más común sobre estas tendencias es que el rápido incremento del número de mujeres jóvenes que reciben educación media y alta coincide con el crecimiento de las actividades burocráticas y comerciales. Además de esto, la gran expansión del sistema escolar y de asistencia a la salud en las ciudades generó un enorme mercado para profesoras y enfermeras. En el mercado de trabajo industrial la mujer perdió importancia relativa en relación al hombre. En vista de ello una proporción importante de mujeres que necesitan empleo son presionadas a recurrir a los servicios domésticos, trabajos menores u otras ocupaciones marginales que ofrecen los más bajos ingresos.

Una de las consecuencias de que las oportunidades ocupacionales se mantuvieran por abajo de las educacionales, es que una minoría de las mujeres que consiguen ingresar a la FT poseen un nivel educacional superior al de los participantes masculinos. "En todos los seis países para los cuales el censo de 1970 permite la comparación, la proporción de mujeres activas que poseen tres años o menos de escolaridad, o analfabetas, es bastante más reducida que las proporciones equivalentes para el hom- 
bre activo. Los porcientos de mujeres activas que poseen 10 años o más de escolaridad son, en todos los casos,, mucho más elevados que los porcientos para los hombres. En una estimación diferente en que se relaciona estrato ocupacional según niveles de educación y sexo, realizada para cinco países, se encontró que en cuatro de ellos las mujeres empleadas en ocupaciones "altas" y "medias" tienen una ventaja educacional significativa sobre los hombres."

La proporción de mujeres empleadas comprendidas en el estrato de salarios más bajo, es mucho mayor que la de hombres. Por otro lado, en relación a los salarios, se señalaron con amplitud otros dos aspectos: se remunera bastante menos a una mujer que desempeña una función idéntica a la del hombre, tendencia que se acentúa en los niveles más bajos. En parte, esto se deriva del hecho de que ella participa en el mercado de trabajo por lo general en un breve período de su juventud, lo cual hace que sus oportunidades de promoción disminuyan y que las consecuentes oportunidades de aumento de salarios sean bastante reducidas.

¿Cuáles serían las expectativas para el trabajo femenino? De manera obvia, la continuidad del proceso de urbanización y burocratización de la sociedad, con la expansión natural de los servicios públicos y particulares, deberá generar un incremento diferencial en la eliminación de ocupaciones no manuales reservadas para la mujer desde hace mucho, además de las de profesora de primaria o secundaria, o de vendedora, etc., y aumentar también las funciones a las cuales se tiene acceso a través de la educación universitaria. La expansión y diversificación de las industrias modernas, la mayoría con tecnología avanzada, deberá significar un campo de trabajo importante para la mujer a pesar de que esto no sea suficiente para compensar las menguantes oportunidades en actividades de pequeño comercio y artesanado.'

En cuanto al futuro de los servicios domésticos, las opiniones son muy divergentes. Los adeptos de la marginalización creciente acreditan que éstos mantendrán su importancia como una de las principales fuentes de ganancias para la mujer. Los datos empíricos sugieren que éstos tienden a perder importancia relativa en el proceso de desarrollo, a pesar de que la parte que subsiste aún sea significativa. ${ }^{110}$

La necesidad de presionar por mayores oportunidades de trabajo para la mujer se considera, a grosso modo, en tres campos de estudio. Para algunos que proponen políticas de desarrollo, la mayor participación de la mujer es un ingrediente omitido con frecuencia que, no obstante, puede dinamizar el proceso. El aumento de la participación femenina en el trabajo es propuesta también en políticas de control de población, con la creencia de que el aumento de este índice debe inducirlas a tener menos

$\therefore$ Op. cit., p. 25.

Este aspecto se aprecia con claridad en Felicia Madeira y Paulo Singer, "Estrutura de emprego e trabalho feminino no Brasil, 1920-1970", Cadernos CEBRAP, 1973. ${ }_{10}$ Paulo Singer, "Emprego e urbanizado no Brasil", Estudos CEBRAP, Núm. 19, 1977. 
hijos. Por último, el trabajo de la mujer es el elemento central para los que propugnan por la igualdad entre los sexos. Conviene agregar, entre tanto, que aún si se tienen en cuenta los tres campos, la preocupación específica respecto al empleo femenino es pequeña en comparación con la atención que recibe la problemática del empleo como un todo.

Las tácticas propuestas para incrementar el empleo femenino,, a grosso modo, acusan y desembocan en proposiciones de alteraciones tecnológicas, y precisamente son criticables por este argumento.

Por su parte, los defensores de la igualdad entre los sexos, que colocan al trabajo fuera del hogar como decisivo para el proceso, la situación en los países de desarrollo dependiente es, por lo menos, irónica. En las sociedades desarrolladas, donde las posibilidades de participación de la mujer en la FT son bastante mayores, si la mujer quisiera competir con los hombres en las tasas de participación en adición a las actividades que realizan fuera de la definición convencional del mercado de trabajo, el problema que de inmediato resulta es el de que ambos sexos asumieran la doble imposición. En América Latina, una parte de las mujeres de niveles de escolaridad "medios" y "altos", pertenecientes a los estratos correspondientes de la población, consiguen resolver este problema, en parte, al echar mano de un servicio doméstico muy barato, desempeñado por otra mujer, lo cual representa en la gran mayoría de los casos una pequeña porción de su salario. Más grave aún es que las mujeres de los estratos más pobres, en la medida en que necesiten obtener empleo para complementar un ingreso familiar inadecuado, son presionadas a realizarlo en adición a su trabajo doméstico y al cuidado de sus hijos, lo cual constituye lo que se convino en llamar la doble jornada de trabajo.

En resumen, se concluye que las mujeres participan en la población económicamente activa, en el estilo del desarrollo dependiente, con sólo un $20 \%$ (en los países desarrollados la proporción gira alrededor del $40 \%$ ), con las peores condiciones de empleo y de salarios. La gran mayoría de ellas, más o menos $53 \%$, permanecen ocupadas en servicios de cuidado de niños y de la casa, y colocadas por este motivo en la categoría de inactivas y no consideradas en estos análisis.

\section{Objetivos}

Nuestro objetivo es analizar el trabajo de la mujer en Fortaleza,, capital de Ceará, ciudad localizada al nordeste de Brasil y una de sus regiones más atrasadas. El análisis se basa en una encuenta realizada por la SERETE Ingeniería y Planeamiento y asesorada en la determinación de la muestra y en el diseño del cuestionario por CEBRAP (Centro Brasileño de Análisis y Planificación) en 1970.

En Fortaleza, se reproducen, a grosso modo, las tendencias recientes observadas en América Latina. El procedimiento con el que se pretende analizar la problemática es diverso. En este análisis, todos aquellos que 
están involucrados en actividades que garantizan el sustento de la población y su reproducción, se considera qué forman parte de la población económicamente activa. Esto significa que entre los económicamente activos se incluyan no sólo a los que reciben remuneración en dinero o en especie y estén involucrados en el modo de producción capitalista —que es el dominante - o en sus modos complementarios — producción simple de mercancía, producción estatal y producción doméstica-, sino también a aquellos que no reciben remuneración y están ocupados en la producción doméstica.

El análisis de la organización de la producción urbana conforme a estas cuatro formas ya ha sido propuesto en otros estudios teóricos. "En lo que se refiere al análisis de la población económicamente activa se conocen dos trabajos que utilizan esta propuesta teórica. ${ }^{12}$

Lo que se pretende esclarecer a través del abordaje adoptado en este trabajo es que el crecimiento de la riqueza, junto a la persistencia también creciente de la pobreza, se deriva de la dinámica que se establece cuando se verifica la penetración y la expansión del capitalismo en un medio en que predominan los otros modos de producción. En lo que respecta al trabajo de la mujer, este abordaje se justifica sobre todo porque el mayor volumen de trabajo de la mujer en los países dependientes se pierde en los modos de producción, cuyo simple volumen denuncia la pobreza de muchos y la riqueza asegurada de pocos.

Son dos los supuestos básicos implícitos de toda la propuesta de análisis: el primero es que una porción significativa de la producción social está constituida por la producción doméstica, y el segundo es la existencia de una relación entre el modo de producción en que están insertas las mujeres y sus características individuales y familiares, en especial su nivel de ingreso monetario, individual y familiar.

Respecto al primero, para entender la incorporación del trabajo doméstico no remunerado, es fundamental esclarecer que no se trata genéricamente de un desarrollo dependiente, sino más bien del desarrollo del capitalismo dependiente, cuyo objetivo no es proporcionar, como pretende hacer creer en cierta forma un tratamiento ideológico - y de esta forma genera expectativas a este respecto-, el creciente bienestar de la población en su conjunto, y hacerla más homogénea, pero sí satisfacer la ley básica de la expansión capitalista: la acumulación de capital. Con base en nuestra proposición de análisis, es fundamental entonces agregar que la esencia del capitalismo es mistificada al nivel de los agentes sociales y que las agencias burocráticas,, las responsables de las medidas oficiales sobre la FT, adoptan esta mistificación. Por último, es esencial señalar que el discurso académico, sea de inspiración marxista o no, adoptó el concepto

"Cf. Vilmar Faria, loe. cit., y Elizabeth Jelin, "Formas de organizacáo da actividade económica e estrutura ocupacional", Estudos CEBRAP, 1974.

I2 Elizabeth Jelin, "La bahiana en la fuerza de trabajo: actividad doméstica, producción simple y trabajo asalariado en Salvador, Brasil, DEMOGRAFÍA Y ECONOMÍA, 1974, 24, Vol. VII. Núm. 3, El Colegio de México; y Paulo Singer, A economía urbana de un punto de vista estrutural: O caso de Salvador, CEBRAP, 1977 (Mimeo.). 
y el sistema de medida oficial, sin percatarse de su permeabilidad a los eventos históricos e ideológicos.

A través de la exploración de este punto principal se inicia el análisis que constituye la primera fase del trabajo.

La validez del segundo supuesto es probada en la segunda etapa, en donde se procura relacionar a las mujeres insertas en los diferentes modos de producción y algunas de sus características individuales y familiares.

Si se dispone de esas informaciones, se está en condiciones de pasar a la tercera etapa, la cual constituye nuestro objetivo final: establecer algunas hipótesis sobre el papel del trabajo de la mujer en la estrategia de sobrevivencia de las familias en Fortaleza.

\section{El concepto de fuerza de trabajo}

Iniciar un trabajo relativo al tema, como se pretende, no constituye ninguna originalidad como lo sabe cualquier persona que haya tenido contacto, aun lateral, con el análisis de la FT. Sin embargo, consideramos que tiene un sentido más profundo de lo que se supone.

Está bastante difundido por una determinada orientación sociológica el compromiso entre una visión teórica dada de la realidad y cuáles y cómo deben ser captados los datos. Cualquier sistema de obtención de datos representa un corte en la realidad que busca revelar ciertos fenómenos considerados fundamentales para el esquema teórico que lo orienta, pero que esconde otros que no son considerados por el mismo.

Esta relación estrecha y viciosa entre teoría e información ha sido enfatizada de manera cada vez más insistente por las ciencias humanas, en la mayoría de los diferentes temas considerados. Es evidente que dentro de esta perspectiva las agencias burocráticas responsables de la captación de datos, de manera inevitable cometen arbitrariedades. No obstane lo que se dice respecto a los datos sobre FT, este tipo de vinculaciones es tan obvia y tan presente que prácticamente todos los estudios en relación al tema, en las diferentes líneas de análisis al igual que las más antiguas, no dejan de enfatizar este hecho. En verdad la crítica va más lejos al denunciar con claridad su compromiso con el momento histórico que lo originó: la célebre crítica al trasplante de conceptos de FT generados en los países desarrollados.

El resultado no podría ser otro, se presta atención al simple hecho de que las informaciones relativas al trabajo de las personas sólo tienen sentido en una sociedad en donde el capitalismo ya ha logrado un grado razonable de expansión. La FT es definida como integrada por el grupo de personas disponibles para participar en la producción social de bienes y servicios. El vínculo con el punto de vista capitalista es claro. El hecho de considerar una población de trabajadores distinta de la población general, implica una organización de la producción en la cual el trabajo se diferencia de otras actividades que satisfacen la propia vida. En las organizaciones de producción preindustrial no es tan nítida esta diferencia. 
Es evidente que no hay una necesidad particular de diferenciar a los que están trabajando de los que no lo están, cuando en la práctica todos los miembros de la familia, de una manera o de otra, están involucrados en la producción de los bienes y servicios necesarios para la subsistencia del grupo.

El párrafo que sigue da una idea de cómo en el capitalismo preindustrial toda la familia participaba del trabajo social.

"Entre las residencias de los patrones están diseminadas en gran número cabanas o pequeñas moradas en las cuales residen los trabajadores empleados, cuyas mujeres e hijos están siempre ocupados,, bordando, hilando, etc., de tal forma que al no haber desempleados, todos pueden ganar su pan, desde el más joven al más viejo. Casi todos los que tienen más de 4 años ganan lo suficiente para su sustento." ${ }^{13}$

Así, es relativamente reciente el interés por identificar, cuantificar y caracterizar la parte de la población que está constituida por trabajadores. Ese interés se manifiesta en un momento en que determinado volumen de individuos son liberados de la industria doméstica, aún cuando haya sido razonable, como resultado del desarrollo de las fuerzas productivas.

Por ejemplo,! antes de 1820 el congreso de los Estados Unidos no veía razón para obtener información sobre los trabajadores del país, a pesar de haber recibido solicitudes de por lo menos dos sociedades culturales para la obtención de tales informaciones antes del segundo censo decenal de 1800. Al igual que en el censo de 1820, la programación preveía el pedido de información sólo sobre el número de personas dedicadas a tres clases de ocupaciones: agricultura, comercio y manufacturas. Este censo de 1820 mostró que existían cerca de 25 millones de personas con ocupaciones especificadas en las tres categorías citadas, de las cuales 2.1 millones estaban en la agricultura,, 349000 (14\%) en las manufacturas y 72000 (menos de $3 \%$ ) en el comercio. El interés por los que trabajaban era lo suficientemente débil para que todas las preguntas sobre ocupaciones fueran simplemente omitidas en el censo de 1830. Las preguntas relativas al trabajo se consolidaron entre 1840 y 1870 . Se puede concluir que el reducido interés por la población que trabajaba, manifestado en los Estados Unidos antes de 1870, no deja de ser una indicación del estancamiento del capitalismo americano. En una sociedad donde la proporción predominante de la población estaba vinculada a la agricultura y en cuyas ciudades predominaban las pequeñas industrias, en las que prácticamente la familia entera participaba como unidad de producción, no podría haber curiosidad o interés por lo que era obvio: casi todas las personas, incluidos los niños, contribuían de alguna forma en la, producción del limitado número de bienes y servicios que caracterizaban el nivel de vida de la época.

El tratamiento conceptual empleado en el censo de 1870 de los Estados Unidos, estableció un padrón para contabilizar a los trabajadores en

\footnotetext{
${ }^{13}$ Daniel Defoe, A Tour thro' the Whole lsland of Great Britain (1724-1726), citado por $\mathbf{P}$. Singer, op. cit., 1976. 
censos subsecuentes hasta 1940. De acuerdo con los resultados censales de 1870, la población ocupada en los Estados Unidos estaba compuesta de personas que informaron tener una ocupación. Un "trabajador remunerado" era una persona mayor a cierta edad especificada (en general 10 o más años) que informaba al entrevistador del censo tener una "ocupación remunerada". Una "ocupación remunerada" era "una ocupación en la cual una persona que se dedica a ella gana dinero o el equivalente en dinero, o en la cual se desempeña en la producción de sus comerciables". Por lo tanto, el supuesto básico es la permuta del desempeño de una ocupación por dinero o algo equivalente. En el fondo lo que de hecho mide el concepto de FT definido así es el avance del capitalismo, o sea cuántos individuos fueron ya incorporados a su tendencia creciente de comercialización y monetarización de las relaciones sociales que tienen como su prototipo la venta de FT transformada en mercancía. Mientras tanto sucede que el fetichismo del mercado y del dinero transforma a los individuos ocupados en actividades que involucran comercialización del producto y remuneración en los únicos responsables de la producción social ubicando, en contrapartida, a los restantes en la situación de dependientes de ellos.

Se divide así a la población en dos categorías básicas: los activos y los inactivos (o sea dependientes) económicamente. Dado que el fundamento es ideológico, la línea divisoria entre lo que es y no es trabajo nunca quedó lo suficiente clara, hecho al que toca la responsabilidad de las frecuentes distorsiones y dificultades de comparación señaladas en los trabajos relativos al tema. Es evidente que cuanto menos desarrollado esté el capitalismo, mayores son las posibilidades de distorsión.

Esta percepción de la realidad es coherente con el papel de las agencias burocráticas. Su función básica es la de hacer mesurables los problemas que se presentan, teniendo que partir para eso de una visión lógica. Sus definiciones terminan por ser fundamentadas en valores convencionales que en general principian con la simple ausencia y presencia de un atributo y su progreso. La visión debería partir "de fuera" de una discusión intelectual. Sin embargo esto ha empezado a ocurrir sólo muy recientemente. El pensamiento científico asumió prácticamente la división impuesta, y el refinamiento del concepto de FT caminó por otra línea todavía en la trayectoria del capitalismo. Lo que se oculta al adoptar este esquema de clasificación se esclarece en el siguiente inciso.

El tipo de orientaciones referidas, utilizadas en los Censos de Estados Unidos, parecían bastante satisfactorias hasta mediados de los años treintas. En el Censo de 1930, realizado en el ápice de la crisis económica, se tomaron las primeras providencias para medir el volumen creciente del desempleo. No obstante, la publicación de las estadísticas de desempleo del Censo generó en mayor medida reacciones críticas que explicaciones. El Censo reveló que había varias clases de desempleados según su naturaleza. 
Si el concepto de FT, medido a través del "trabajador remunerado" se vincula a la expansión del capitalismo en un área, el concepto que expresa implica que las nociones de empleo y desempleo se vinculen a situaciones de crisis del capitalismo industrial avanzado.

A partir de 1940 se empezó a utilizar un nuevo marco conceptual en los Estados Unidos. Se agregaron dos elementos esenciales a la nueva orientación. En primer lugar, el concepto de FT dejó de referirse al status (poseer una "ocupación remunerada") y pasó a considerarse como un concepto de cuño "circunstancial", cuyo contenido se refiere y está basado en una actividad,, de manera específica la actividad de trabajar y proporcionar trabajo. El abordaje de "ocupación remunerada" no precisaba por ejemplo si el individuo aún estaba trabajando en aquella ocupación o si ya se había retirado. También quedaban fuera de la FT las personas que nunca habían trabajado y que no poseían por eso ninguna ocupación, en especial aquellos que nunca fueron adiestrados para ningún trabajo específico. En segundo lugar, el concepto requirió un período de referencia determinado: actividad durante una semana específica, o bien la semana que precede a la entrevista. A través de esta nueva orientación la población que trabajaba se convirtió en población ocupada o que buscaba trabajo durante una semana específica. El objetivo era entonces medir la disponibilidad real para el trabajo.

Los países de menor desarrollo relativo empezaron a utilizar estos conceptos para estimar su FT. La gran mayoría escogió una u otra, o ambas, exactamente como fueron descritas aquí o con pequeñas variaciones. $\mathrm{Mu}$ cho muy pronto se empezó a ver con claridad que ambas orientaciones presentaban serios inconvenientes para estimar la FT en tales países. En la práctica las críticas se concentraban en torno al trasplante de los conceptos basados en la experiencia de regiones de industrialización avanzada.

En cuanto al procedimiento de "ocupación remunerada", según la mayoría de los autores la inadecuación se derivaba de suponer una economía del todo monetaria, en la cual una gran proporción de FT estaba constituida por empleadores en una relación contractual. Ésta no es la situación en los países de industrialización reciente, donde todavía una gran parte del trabajo agrícola es de subsistencia y en las ciudades el pequeño comercio y las manufacturas con frecuencia recurren aún al trabajo familiar no remunerado. En cuanto a la segunda orientación, la crítica fundamental se refiere a la medida del desempleo. Como se observó, el método consiste en contar a las personas sin trabajo y que de hecho buscaban trabajo en un determinado período de referencia. Lo que de hecho se mide según esta conceptualización es el desempleo visible toda vez que si persiste una elevada tasa de desempleo, es bastante probable que simplemente las personas renuncien a buscar trabajo. De este modo, determinado volumen de desempleo visible debe ir acompañado por un volumen mucho mayor de desempleo invisible, el cual es prácticamente imposible de estimar. En realidad, para que una persona se disponga a buscar empleo es necesario que haya una perspectiva de encontrarlo. Por este motivo, este concepto se 
aplica en los países en donde el desempleo es cíclico. Con certeza ésta no es la situación de nuestros países, donde el desempleo es en apariencia crónico y los índices de desempleo visible sólo pueden estar muy por abajo de la realidad.

Mientras tanto, ésta mostró no ser la mayor dificultad para apreciar la problemática del empleo. A ésta se sumó el problema del subempleo. Así como la inclusión del desempleo en la medida de la FT se refería históricamente a la crisis del capitalismo, la medida del subempleo se refiere a la crisis del capitalismo dependiente, descrita en la primera parte; por otro lado, el mismo concepto de subempleo cambió de manera sustancial en los últimos años.

Al principio se definían como subempleados aquellos que poseían un empleo pero que desearían trabajar más. Quedó luego mucho más claro que la evaluación del desempleo era arbitraria según esta definición. Según el límite respecto al que se considere el empleo y el subempleo, se puede obtener un mayor o menor volumen de subempleo dado que éstos son complementarios.

Para los economistas la categoría subempleo tiene otra connotación. Estaban desempleados aquellos individuos cuya capacidad no se utilizaba con eficiencia trabajando o en tiempo parcial o con jornada normal. Subempleados serían los individuos que podrían ser eliminados si la tarea que efectuaban se organizara de otro modo.

Como no se puede medir de manera directa la productividad de cada ser humano, se intentó hacerlo en forma indirecta evaluando sus respectivos salarios. En este caso, la medida del subempleo no se distingue prácticamente de la medida de la probreza, sin contar que la arbitrariedad persiste.

Más recientemente se acrecentó la duda en cuanto a la validez de las definiciones de la situación del subempleo. Se refiere a su "compromiso con el modelo teórico orientado al sector moderno y más avanzado de la economía". De manera fundamental, lo que se argumenta es que el modelo general supone una homogeneidad tecnológica en las formas de organización de la producción, que no existe en las regiones subdesarrolladas. Al contrario "en estas regiones existe una heterogeneidad tecnológica y de modos de producción que fundamentan categorías ocupacionales, formas y niveles de subutilización específicos". ${ }^{14}$ Así, las características del subempleo no son sólo personales y sociales, como las descritas, sino también "las determinadas por el modo de inserción del trabajador al sistema productivo". ${ }^{515}$ Esta forma de enfrentar el problema ha conducido a desarrollar una forma alternativa de analizar la subutilización de la mano de obra inserta en los estratos tradicionales y donde "predominan relaciones de producción atrasadas".

${ }^{14}$ P. M. Hauser, "Forca de trabalho", Hand Book of Modern Sociology, Faris Ed., Chicago Rand Nc Nally, 1964.

$$
\text { Cfr. IPEA, op. cit. }
$$


Veamos ahora cómo repercutió la evolución del concepto de FT en la medición del trabajo femenino.

\section{EL CONCEPTO DE FUERZA DE TRABAJO Y EL TRABAJO DE LA MUJER}

Al igual que en cualquier estudio dedicado al análisis del trabajo femenino en los países de desarrollo dependiente, se tienen precauciones respecto a las limitaciones de las conclusiones en virtud de las distorsiones derivadas de conceptos y técnicas utilizadas por los agentes oficiales sobre la medición de la FT. En su gran mayoría la explicación de las distorsiones gira, de la misma manera, en torno a lo siguiente - en general el concepto de FT supone una economía monetaria en la cual la mayor parte de la FT está constituida por empleados con una relación contractual. Sucede que en estos países la mayor parte del trabajo femenino se concentran en actividades que aun cuando sean extrafamiliares,, se desenvuelven dentro de los límites del domicilio. Las mujeres que se encuentran en esta situación, si son clasificadas como ocupadas permanecen de esta forma dentro de la FT, y si son clasificadas como desocupadas quedan entonces fuera de la FT en la categoría de inactivas.

El volumen de FT femenina se altera de manera sustancial si se opta por uno u otro criterio. Boseruji ${ }^{16}$ muestra que como este sesgo se refiere a trabajos preindustriales, la tendencia de las tasas de participación femenina siguen caminos diametralmente opuestos cuando se acelera el proceso de industrialización de la economía, si un país opta por uno u otro criterio.

Si la mujer involucrada en actividades que combinan dentro de su domicilio trabajo doméstico y extradoméstico fue incluida en la FT, la tasa de población femenina económicamente activa tiende a aumentar con la expansión de la industria y de la mecanización en general; en caso contrario la tasa tiende a disminuir.

Aun cuando las distorsiones hayan logrado tal nivel, se hace muy poco, en términos comparativos, con toda la atención que concentró la problemática del concepto en la tentativa de desentrañar la naturaleza de este sesgo. Lo sorprendente es la claridad con que este sesgo se deriva, en esencia, del hecho de que los agentes burocráticos involucrados en el proceso de recolección de datos asumieron, más o menos, la ideología que propala que la función principal de la mujer en la sociedad es el trabajo doméstico.

Sin embargo, el compromiso ideológico es anterior. Los individuos involucrados en actividades para el consumo de la familia —realizadas en el ámbito doméstico-, las cuales casi en su totalidad son mujeres, son

\footnotetext{
${ }^{16}$ Esther Boserup, "Employment of Women in Developing Countries", International Population Conference, Lieja, 1973, Vol. 1.
} 
excluidas de la población económicamente activa. La verdad es que un análisis un poco más preciso de la definición de dependientes muestra de manera casi transparente una falta absoluta de criterio claro y objetivo para excluir a las mujeres de esta categoría y sugiere por sí solo, lo cual nosotros avalamos, la necesidad de una versión explicativa de la ideología del problema. ${ }^{17}$

La hipótesis más inmediata es que la exclusión se origina en la no remuneración. Como ya se comentó, en la economía capitalista existe una tendencia creciente a la monetarización de las actividades que tienen como modelo la venta de la mercancía FT en el mercado. Así, debido al encubrimiento, a través del fetichismo del precio, cualquier actividad que no fuese cambiada por dinero de manera automática dejaría de ser económica. Sin embargo, este criterio no se aplica a otras categorías de la población. Por ejemplo, no se excluye al campesinado de subsistencia, que aún constituye una parte sustancial de la FT en los países de industrialización tardía, o a los miembros (en especial los masculinos) de la familia rural o urbana que trabajan sin remuneración. El criterio utilizado para su exclusión no es tampoco el mercado, porque si se utilizara excluiría al agricultor que siembra sólo para su autoconsumo. Faltaría considerar sólo, como última alternativa, la naturaleza del trabajo. Todo indica que éste no es tampoco el criterio, dado que la empleada al igual que el ama de casa, o el chofer que desempeña el mismo tipo de actividad por una remuneración en dinero o en especie, generalmente se considera como trabajadora y de esta forma parte integrante de la FT.

Nuestra hipótesis es que la exclusión de la mujer que realiza trabajos domésticos para consumo de su familia se liga a un fetichismo más del capitalismo utilizado para enmascarar el precio de la reproducción y reposición de la FT. El carácter real de la producción capitalista, el origen de la plusvalía, es obscurecido de manera consciente por los agentes, lo que hace que el trabajador vea en) su salario el precio de su trabajo y no el precio de manutención y reproducción de su FT. Con los mismos objetivos, se institucionaliza la fantasía de que los bienes necesarios para la subsistencia del trabajador y su familia son obtenidos totalmente a través de los salarios. Al clasificarse el trabajo doméstico como un no trabajo, se oculta el hecho de que un trabajo adicional fuera del circuito monetario del sistema es un elemento importante para la propia supervivencia del trabajador y sus dependientes.

Lo que sorprende, pero que al mismo tiempo es un argumento en favor de la fuerza y naturaleza ideológica que permea el fenómeno, es que la desmistificación del trabajo doméstico, o sea la aceptación del papel productivo de la mujer en esta condición dentro de la familia,, y jeconómico en la sociedad, sea tan reciente, no obstante la evidencia de que las unidades domésticas constituyan además de unidades de consumo también unidades productivas.

${ }^{17}$ La falta de objetividad fue señalada antes por P. M. Hauser, op. cit., y Jelin. Cfr., op. cit., 1974. 
Los bienes adquiridos en el mercado no pueden ser consumidos antes que un trabajo adicional los haga útiles para el consumo final. Preparar la comida, mantener la limpieza de la casa, realizar la confección de ropas y objetos, etc., constituyen diferentes formas en las cuales se concretiza el trabajo doméstico.

La familia y la unidad doméstica han sido analizados de manera tradicional como si desempeñaran funciones casi idénticas, que serían de naturaleza doble: biológica (manutención y reproducción de los miembros de la sociedad) e ideológica (socialización de los niños). Es comprensible, o por lo menos consistente, que los análisis que se hacen al nivel de la coherencia de las manifestaciones de los fenómenos, como es el caso de las teorías que orientan los conceptos utilizados por las agencias burocráticas asuman esta postura. Mucho más complejo es intentar comprender por qué análisis que se proponen desentrañar los procesos que originan las instituciones acaban por adoptar tal conceptualización. Durante mucho tiempo, sin insistir tanto en este tema, los análisis que propugnan por utilizar una postura marxista, atribuían a la familia, en esencia, la función de manutención y reproducción de la fuerza de trabajo y también la tarea ideológica de transmisión de valores tales como individualismo, sexualidad, autoritarismo, fundamentales para la manutención de la clase trabajadora y la aceptación, por parte de ésta, del capitalismo. Se le atribuían también funciones de naturaleza psicológica, en especial respecto a las compensaciones para el individualismo.

Por cierto que la familia o la unidad doméstica, incluido en este caso el lugar donde especialmente se une, desempeñan papeles ideológicos y psicológicos estratégicos para la preservación del sistema, inclusive por la flexibilidad de adaptación a las nuevas situaciones. Esto no elimina que también posea funciones económicas igualmente estratégicas y flexibles en la sociedad capitalista.

En las sociedades precapitalistas, la familia se confundía con una unidad básica de producción, y como tal la producción general se agotaba prácticamente en los límites del domicilio. El capitalismo trazará una alteración fundamental para la posición del trabajo doméstico dentro del trabajo general. A medida que el capitalismo se expande, la unidad doméstica se especializa cada vez más en la producción para el mercado de la mercancía "fuerza de trabajo", adquiriendo para esto mercancías producidas en las unidades capitalistas, eri donde se concretiza la plusvalía. ${ }^{18}$ Es indudable que existe una tendencia en dirección a la polaridad descrita; sin embargo, esta situación límite nunca se concretizó ni se puede concretizar como se verá más adelante.

Desde la perspectiva de un análisis marxista, lo que se argumentaba para impedir que los individuos ocupados en tareas domésticas no remuneradas fuesen incluidos entre los que participaban en la producción social, era su

${ }^{18}$ Walli Secombe, "The House Wife and her Labor under Capitalism", New Left Review, Núm. 83, enero-febrero de 1974. 
aparente desvinculación total con la ley del valor, o sea con la apropiación de la plusvalía. ${ }^{19}$ Lo interesante es que no se excluyera a los individuos ocupados en actividades relativas a la producción estatal o producción simple de mercancías, que tampoco genera plusvalía, para no mencionar a la agricultura de subsistencia. Las relaciones entre el proceso de acumulación - cuyo móvil es la plusvalía y las formas no capitalistas de organizar la producción - son claras si se tiene en cuenta que las mismas no son desempeñadas dentro del mundo capitalista pero sí desarrolladas bajo relaciones de producción capitalistas,, o sea, al distinguir el modo de producción capitalista del capitalismo como un sistema en el cual el modo de producción capitalista es el dominante pero no el único ya que se vale de otros modos de producción subordinados. ${ }^{20}$ El modo de producción dominante es el que determina la estructura y el movimiento total de formación social. Cuando un sistema económico es caracterizado como capitalista, significa que el modo de producción dominante es el capitalista y que los diferentes modos de producción están articulados bajo su mando y en función del ritmo de la acumulación de capital. Esta misma línea de argumentación se puede utilizar para incorporar la producción doméstica en el cómputo de la producción social.

De manera más clara, el sentido de la exclusión del trabajo doméstico, en especial en el caso de los países no desarrollados, en su relación con la explotación capitalista es preciso especular un poco más y ver qué ocurre cuando el capitalismo se expande en un medio en que predominan otros modos de producción. La hipótesis más moderada y que se menciona aquí es la de Singer." "El capital penetra en determinadas ramas de actividad, donde posee mayores ventajas en relación al modo de producción preexistente, revolucionando los métodos de producción e introduciendo otras relaciones de producción. Mientras tanto, éste surge mediante la implantación de actividades nuevas que sólo él es capaz de suscitar. De todos modos, el resto de la economía no se afecta de manera inmediata, permanece con su estructura anterior. Se genera entonces una interrelación doméstica entre la economía capitalista y los otros modos de producción, que se ponen por así decirlo al servicio del capital. Algunos se transforman en soporte financiero del modo de producción capitalista, otros en depósitos de mano de obra y otros aún en proveedores de bienes y servicios complementarios para la producción capitalista".

Así, es posible distinguir cuatro modos de producción en una economía urbana del tipo de la que queremos estudiar.

\footnotetext{
19 Ibid.

John Harrison, "The Political Economy of House Work", Bulletin of the Conference of Socialist Economist, invierno de 1973.

P. Singer, he. cit.
} 


\section{Modo de producción capitalista}

La economía de las ciudades, aun cuando no sea el modo que absorbe al mayor número de población económicamente activa, puede ser caracterizada capitalista en el sentido de que es dominada por el modo de producción capitalista. "El capitalismo se caracteriza por la propiedad privada de los medios de producción, lo que confiere a la clase capitalista el comando del proceso de producción mientras que su ejecución está a cargo de los trabajadores asalariados. La producción capitalista adopta siempre la forma de mercancía y el valor excedente (plusvalía) es apropiado por los capitalistas, parte del cual es acumulado bajo la forma de nuevo capital. La ley básica de la dinámica de este modo dé producción es que su expansión depende del ritmo de acumulación de capital, o sea de la reinversión de ganancias."

"Lo que ya define á la producción simple de mercancías y lo diferencia de la producción capitalista, es el hecho de que el dueño de los medios de producción participa de manera directa en el proceso productivo. En principio, el productor directo es entonces un agente autónomo, pero puede disponer eventualmente de auxiliares asalariados. El productor autónomo pasa a ser un empleador, o sea un administrador del capital, y la empresa pasa a formar parte del modo de producción capitalista cuando la expansión de la empresa alcanza un nivel en el que el propietario no dispone más de tiempo para participar en la producción y pasa a ejercer sólo funciones de dirección y de supervisión. La producción simple de mercancías predomina en actividades cuya organización en gran escala por el capital no presenta ventajas claras. En la medida en que el productor autónomo abarata su producto, depreciando el valor de su trabajo y recurriendo a las ganancias de sus inmuebles, interesa al capital que determinadas actividades de que se sirven continúen organizadas en el modo de producción simple de mercancías. En otros términos, , los precios de las mercancías producidas por ellos, apenas cubren sus costos de producción, sin incluir un margen de ganancia proporcional al valor de los medios de producción".

Las productoras simples de mercancías incluyen fundamentalmente las artesanías de encaje, costureras, bordadoras, etc., y las que prestan servicios personales no establecidos-lavanderas, planchadoras, etc. Esos trabajos en general son realizados dentro de la morada por mujeres sin especialización, que no pueden dejar sus casas debido a los hijos pero que necesitan de una fuente de ingreso y tienen como única opción la comercialización de un excedente de trabajo doméstico.

En el fondo, como no hay necesidad de bienes propios para la producción, poder recurrir a la mujer constituye una fuente de ingreso siempre que no tenga otra posibilidad de empleo, ya sea por sus propios problemas o por el lado del mercado.

Las mujeres que producen servicios personales no "establecidos" y las

\footnotetext{
${ }^{22}$ Las características de los modos de producción son reproducciones exactas a los extractos hechos por P. Singer. loe. cit.
} 
artesanas realizan estas tareas como complemento de su actividad doméstica y cobran por ellas muy poco. La situación cambiará cuando la empresa capitalista considere conveniente abarcar la industria de confecciones y prestación de servicios, lo cual debe ocurrir cuando el nivel de vida de amplios sectores de la población se eleve, o que dependa en última instancia,, de la reserva de mano de obra.

"Pertenecen a la producción estatal las actividades del poder público (financieras, fiscales, estadísticas del gobierno, policía, fuerzas armadas, tribunales, penitenciarías, etc.) y las actividades sin fines de lucro (educacionales, de asistencia social, sindicales, políticas) realizadas por entidades estatales, religiosas, asociaciones, etc. Se trata en último análisis de producción de valores de uso para consumo colectivo o social. Son servicios que, o no pueden por su propio carácter ser vendidos o, cuando se puede hacerlo, encarecerán de manera excesiva la reproducción de la FT. La socialización de los servicios para los cuales existe una demanda solvente, de manera notable en el caso de la educación, salud y previsión social, constituye una especie de subsidio al capital, que puede así dejar de pagar salarios que cubran del todo los costos de reproducción de la FT que explota."

"La producción doméstica se distingue de manera fundamental de la producción simple de mercancías, en especial las realizadas por las mujeres, no por el local de trabajo sino por el destino de su producto que es consumido en el propio círculo del hogar. Hace que parte de la producción doméstica, la actividad del ama de casa (que cocina, limpia objetos, repara, lava y plancha la ropa, hace compras, cuida de los niños pequeños,, etc.) e incluso la de otros miembros, lo mismo que los masculinos, colaboren también fundamentalmente en la construcción, expansión y manutención de la morada."

La cantidad de trabajo doméstico varía de manera sustancial. Varía no sólo en función del grado de desarrollo del capitalismo, de la división y especialización del trabajo, sino también, dentro de una misma formación social, entre las diferentes clases sociales.

En las sociedades con elevado nivel de industrialización, el trabajo gastado en la producción de bienes y servicios para el consumo familiar es reducido por dos motivos que resultan del mismo proceso: el elevado nivel de vida logrado por la población de tales países. En primer lugar, éste disminuye porque gran parte de los bienes producidos antes en los límites domésticos se vende en el mercado y, en segundo lugar, porque la utilización de aparatos domésticos disminuye el tiempo para producirlos.

En las áreas subdesarrolladas las familias de ingresos altos, que en teoría podrían limitar al mínimo el servicio doméstico, utilizando servicios del mercado y electrodomésticos, no lo hacen porque el servicio doméstico asalariado permite mantener, con costos relativamente bajos,, un nivel considerable de producción doméstica. La utilización de trabajo doméstico asalariado en la producción doméstica, hace que ella pierda su carácter no mercantil pero no se contrapone su esencia respecto al destino de la pro- 
ducción que continúa siendo para el autoconsumo. La base material de la producción doméstica está constituida por los salarios recibidos por los miembros del domicilio ocupados en otros modos de producción. La extensión del trabajo doméstico debe oscilar en función del ingreso que entra al domicilio. Mientras tanto, es evidente que si las ganancias monetarias disminuyen por abajo de cierto nivel mínimo, la contribución del trabajo doméstico se torna insuficiente y otros miembros estarán obligados a buscar trabajo a la vez que comprometen la reproducción normal de la fuerza de trabajo.

Aun cuando difieran en cuanto a la extensión y su carácter mercantil, los efectos de la producción doméstica son los mismos. El volumen del trabajo doméstico es tanto mayor cuanto mayor fuera el interés por mantener bajos los niveles salariales y sin contar con la mayor oferta posible de mano de obra.

La existencia de un servicio doméstico relativamente barato y abundante incide sobre todo en la calidad de vida de las clases dominantes, cuyo capital es más bien reducido en cuanto a sus similares en los países desarrollados, en tanto no disfruten de un nivel de vida igual o mejor. Esto sucede por dos motivos: por la economía de capital y por la posibilidad de concentrar el capital en actividades industriales y conexas.

La subsistencia y la reproducción de la FT, al igual que en una economía típica capitalista, está por lo tanto bastante lejos de concretarse a la recepción de salario originado de la explotación capitalista y su gasto en la adquisición de mercancías producidas de esta manera. En este proceso participan relaciones de producción no capitalistas que pueden variar en extensión, pero que son fundamentales para el capitalismo. La mercancía no es producida sólo de manera capitalista, se realiza aún como una producción de valores de uso, individuales o colectivos, sin los cuales los salarios deberían ser bastante más elevados para cubrir plenamente los costos de la "mercancía FT".

Por lo tanto, la producción está organizada según la orientación del modo de producción capitalista pero que subordina otros modos en su favor. Los modos de producción difieren en esencia en relación a la propiedad o al control de los medios de producción y del destino del producto por lo que pueden parecer en algunos aspectos y diferir en otros. Por ejemplo, con relación a la propiedad la producción doméstica se asemeja a la producción simple de mercancías. Con relación al destino del producto en cierto aspecto se aproxima a la producción estatal en la medida en que ésta produzca valores de uso y no mercancías, a pesar de que sean para consumo colectivo y no individual.

Nuestra hipótesis es que algunos aspectos específicos de la producción doméstica son fundamentales para el sistema capitalista y por eso fueron construidos de tal forma que la hacen en extremo maleable para manipulaciones ideológicas, adaptable como un guante a las conveniencias del capital.

La producción doméstica no es un modo de producción aislado como 
los otros; está acoplado a todos los otros modos de producción. Es en el ámbito de la producción doméstica donde se reproduce lá fuerza de trabajo explotada por el capital mientras que la reproducción de la capacidad de los trabajadores que están comprometidos en la producción estatal y en la producción simple de mercaderías ocurre de igual modo en la producción doméstica. Ésta se liga por lo tanto de manera orgánica a los demás modos de producción, con lo cual constituye una base común del proceso de reproducción social. El hecho de que la producción doméstica sea la única en producir y mantener biológicamente a los individuos que componen la sociedad y también en inducir valores socialmente aceptados y dar apoyo emocional, la hace en extremo flexible para que se atribuya únicamente a ella esta función.

Los individuos considerados más aptos para realizar la producción doméstica no se distinguen por habilidades adquiridas o selectas, sino porque por tradición desempeñaron aquellas funciones y pertenecen de manera natural a una organización doméstica determinada. De esta manera, fue relativamente fácil transformar una actividad que por tradición fue desempeñada por mujeres en la principal actividad de la mujer, así tenga necesidad o no de desempeñarla.

Por último, esta hipótesis adquiere mayor consistencia si se agrega que "al contrario de lo que indican las apariencias, el capital no explota recursos naturales o mercados, sino trabajo social. Es éste el que se convierte en valor, parte del cual es acumulado bajo la forma de capital nuevo". ${ }^{23}$ De ahí el interés por enmascarar el carácter y extensión de su explotación.

No obstante que la producción doméstica y la producción simple de mercancías, en especial las típicamente femeninas, sean actividades que tienden a disminuir con la expansión del capitalismo, pueden funcionar siempre como una alternativa cuando el capitalismo entre en el ciclo decreciente $\mathrm{y}$, en consecuencia, se agranda el ejército industrial de reserva. De este modo, la producción doméstica y la producción simple de mercancías "femeninas" se integran subordinadas al "modo capitalista" dominante, que las utiliza en función de la acumulación de capital con todo y que a veces está más interesado en auxiliarlo y reproducirlo que en su desaparición. Por supuesto que recurrir a este expediente es más fácil y su función mistificada de manera más fácil en cuanto a mantenerse una definición social de tarea doméstica como ámbito privilegiado de actividad de la mujer.

El considerar la actividad doméstica como reservada a las mujeres, no sólo hace más fácil escamotear el papel de esta actividad en el proceso de acumulación de capital, sino también la naturaleza diferente del trabajo doméstico de las mujeres de las diferentes clases sociales. Dentro de la misma categoría se considera como "ama de casa" tanto a la mujer del operario como a la del capitalista, aunque no haya más diferencia que la naturaleza del trabajo desarrollado por cada una de ellas. ${ }^{24}$

${ }^{23}$ P. Singer, "Emprego, producáo e reproducao de forja de trabalho".

24 Chico Oliveira, "Trabalho feminino e riqueza capitalista in o banquete e o sonho", Cadernos de Debate, Ed. Brasiliense, 1976. 
DEMOGRAFÍA Y ECONOMÍA XII: 1, 1978

Por último, características propias de la organización doméstica permiten ocultar la disponibilidad real de FT femenina, o sea del ejército industrial de reserva cuando se utilizan los conceptos convencionales de FT.

\section{EL CONCEPTO DE DESEMPLEO Y DEL TRABAJO DE LA MUJER}

En general, el objetivo de los estudios de FT es medir su grado de inserción en el proceso de producción social. Los que no consiguen su incorporación son denominados desempleados. Tal propósito sólo tiene sentido en una sociedad capitalista ya que sólo en ella se propicia taltipo de desempleo. Así, en una unidad de producción capitalista la inversión del individuo en la división del trabajo tiene como finalidad su contribución a la formación del excedente social. El número de trabajadores que consiguen incrustarse en la división del trabajo está compuesto por aquellos cuyo producto contiene más valor que el de su FT. Este número está determinado entonces por condiciones independientes, por lo menos de manera indirecta del volumen de oferta de FT. Por lo tanto existe siempre la posibilidad de que el número de empleados efectivamente sea menor que la oferta de FT; en otras palabras, que haya desempleo.

Por tradición las actividades domésticas han permanecido a cargo de las mujeres residentes en las unidades familiares. A diferencia de las unidades productivas capitalistas, ellas se encargan del trabajo doméstico como consecuencia directa de ser miembro de aquella unidad de residencia. En estas circunstancias, en una unidad doméstica nunca aparecería un desempleo involuntario, entendido éste como la existencia de individuos imposibilitados de participar de la división de trabajo de la unidad, sólo por razones económicas. En esta organización, cuando hay un trabajador adicional por lo general existe una redistribución de tareas. Así, lo que puede ocurrir dentro de una organización doméstica es un empleo disfrazado. Esto significa que hay un cierto número de productoras excedentes cuya contribución para aquella organización sería nula o negativa a pesar del hecho de que estas mujeres participen de la producción de la unidad.

Medir el trabajo excedente en una unidad doméstica es sin duda bastante complejo. En el fondo, la capacidad productiva de las mujeres tiende a confundirse con la propia demanda de bienes y servicios domésticos cuya extensión, como se observó antes, puede variar de manera sustancial.

La subutilización de una mujer que realiza tareas domésticas es más evidente si la mujer tiene alguna especialización o puede optar por otra actividad, o sea si fuera evidente su alternativa en el mercado de trabajo.

Por tradición, los modelos utilizados para medir la población disponible, comprometidos con la óptica y la problemática del capitalismo, naturalmente en nada han contribuido para evidenciar este tipo de subutilización. Al contrario,, ésta ha mostrado ser una situación bastante propicia para ocultar la reserva de trabajo femeinino, dado que con frecuencia hay oportunidad de que se intercale de algún modo una mujer más en la pro- 
ducción doméstica y por ese motivo se considere ocupada en esta actividad y por lo tanto fuera de la FT O inactiva.

Si el criterio fuera la "ocupación principal" u "ocupación remunerada", la falta de una especialización la coloca fuera de la FT.

Si el criterio de determinación de la tasa de participación se basa en la manifestación de la voluntad de trabajar, todo dependería de las posibilidades ofrecidas por el mercado de trabajo. Este concepto supone, como ya se observó, una visión optimista del mercado de trabajo, situación que con certeza no corresponde сод la de los países subdesarrollados. En el caso del desempleo generalizado, no es de extrañar que exista un sector considerable de personas que con deseos de trabajar y disponibles para ello, opten por no participar, con lo cual acreditan que sus posibilidades son prácticamente nulas. En esta situación las mujeres son también clasificadas como inactivas. Sin embargo, en realidad en ambas situaciones son desocupadas ocultas.

En este trabajo se busca identificar a las desocupadas ocultas. Es obvio que no se espera determinar la magnitud del fenómeno, tarea en extremo compleja. Se tiene en cuenta también que cualquier criterio implicaría introducir un elevado grado de subjetividad y que por lo tanto sería arbitrario.

Lo que se hizo fue analizar cada cuestionario en forma individual, con el supuesto implícito de que las desocupadas ocultas serían las mujeres que de manera convencional se clasificaran como inactivas, pero que poseían capacidades potenciales semejantes a algunas que serían consideradas como activas. Se consideró como referencia en especial la existencia de otra persona disponible para realizar el trabajo doméstico así como la edad y educación de las mujeres.

Como se podría argumentar, dados todos los supuestos implícitos, tal fenómeno no tiene una importancia sólo académica. Por el contrario, revela una faceta más del fetichismo que envuelve el significado del trabajo de la mujer, ya que a pesar de que adopte proporciones mayores en los países de menor desarrollo relativo, no deja de existir en los países desarrollados.

Como justificación de la tarea realizada, se considera que vale la pena adelantar una conclusión. En lo que se refiere a las bandas de ingresos más bajos, todo indica que el trabajo doméstico sirve de máscara al desempleo oculto y que a medida que se pasa a niveles más elevados de ingreso, la categoría de "estudiante" parece adaptarse mejor.

\section{El trasplante de conceptos}

Como se mencionó antes, la crítica de la mayoría de los autores que escriben sobre el tema de la FT, se concentra en el transplante de conceptos originados en países de industrialización avanzada, al contexto de los países subdesarrollados. En estos términos, existe en el fondo la convic- 
ción de que estos conceptos siempre estuvieron muy bien adaptados a la realidad que se procuraba medir.

Nos parece que todo lo expuesto sugiere que esto no es verdad y que la crítica tiene otro punto de partida. En primer lugar es preciso recordar que el trasplante, desde el punto de vista de la operación, es sin duda legítimo y parece natural el "aprovechar" una metodología existente. La crítica que se hace al trasplante de conceptos sin duda alguna es procedente, pero el concepto no deja de explicar la realidad del subdesarrollo sólo porque es trasplantado, sino exactamente porque el marco teórico que lo orienta considera legítimo este trasplante. Por lo tanto, lo que debe ser criticado es el propio marco teórico utilizado.

Lo que se pretendió mostrar fue que los agentes burocráticos partieron de una polarización que no sólo es arbitraria sino sobre todo ideológica, que al mantenerse al nivel de la coherencia del sistema, aisló al trabajo doméstico de la producción social. Obscureció así una faceta de la propia esencia de la explotación capitalista.

Esta división nunca fue puesta en duda por los responsables de los informes oficiales. El paso siguiente fue dado después de la crisis de los 30, y originó una orientación conocida como "fuerza de trabajo" comprometida con la fantasía keynesiana de desempleo involuntario. Sin duda es pertinente mostrar que este modo de abordar el tema sólo se explica o, más bien, sólo se aplicaría a economías cuyas instituciones se aproximan a las implicadas en el modelo keynesiano y cuyas autoridades estaban por lo tanto interesadas en obtener informaciones apropiadas para los problemas que permite analizar tal modelo. En una sociedad con desempleo crónico no se podría pretender conocer su problemática a través de este modelo.

Mientras tanto, como se mostró en el inciso I I I, sucede que el concepto de subempleo, a pesar de que no pecase por el hecho específico de ser transplantado de los países desarrollados que sufrieron la crisis de los años treinta,, pero originado en la tentativa de solucionar nuevas crisis propias, mostró una impresición tan problemática para el contexto subdesarrollado como el concepto de desempleo abierto. Las distorsiones se derivan de su compromiso con el sector capitalista, por estar penetrado de la tradición keynesiana, a pesar de que éste sólo se refiera a una medida del desempleo.

El problema no radica entonces en traducir a Keynes, sino en no tener en cuenta toda una tradición de literatura que señala el hecho de que el sistema capitalista tiene un "modo de producción capitalista" como dominante y modos de producción complementarios subordinados a él en función del ritmo de acumulación del capital. El trabajo excedente en estos modos es el resultado de la combinación de leyes propias de formación de excedente de trabajo de cada modo y su relación dinámica con el modo dominante.

Como se observó antes, éstas constituyen las tentativas más recientes de medir la subutilización de la FT. ${ }^{25}$ Mientras tanto, se hizo caso omiso del 
"modo de producción doméstico". Según nuestra hipótesis estos análisis fallan por no considerar que determinadas actitudes, con relación al trabajo de la mujer, son moduladas por necesidades y ventajas del capitalismo al institucionalizar el papel económico del hombre como suficiente para mantener a la familia y el papel de la mujer como simple consumidora.

Es obvio que de todo lo dicho no se debe concluir que los Censos deben ser suprimidos, pero sí que son permeables a los eventos históricos e ideológicos y que una propuesta de análisis académico debe tener en cuenta este hecho. $\mathrm{O}$ como señala $\mathrm{Cicorrel}^{26}$ "en adición a los significados culturales y de lenguaje, los sociólogos deberían intentar poner en evidencia el sentido de la propia medida que utilizan". Ésta no es una tarea fácil, numerosos conocimientos e interpretaciones que se utilizan en el montaje de los datos invariablemente se pierden, con lo que sólo queda para el usuario la forma cuantitativa.

Un ejercicio realizado como intento de percibir más allá de los datos resultó bastante interesante. El Censo demográfico de 1950 subestimó en gran medida el número de mujeres y niños que ejercían sus actividades en sus domicilios, tanto en las zonas agrícolas como en la ciudad. El origen de la subestimación quedó muy claro cuando se analizó el manual distribuido a los entrevistadores y en el cual se señala:

"En cuanto a la explicación principal, es conveniente aclarar que ciertas ocupaciones, aun cuando no remuneradas, pueden constituir la principal actividad de los entrevistados. Muchas "amas de casa" que de hecho ejercen, cómo ocupación principal, la dirección de la casa, pueden declarar, por error, que tienen como ocupación principal otra actividad cualquiera sólo porque en las horas en que sus labores domésticas lo permiten, ganan algún dinero al ejercer otra actividad".

Ya nos referimos a la tendencia del capitalismo a producir, cada vez más, los bienes y los servicios necesarios para la sobrevivencia del trabajador en el mercado, y por lo tanto fuera de la unidad doméstica, hecho este que tiende a ocurrir en forma paralela con la mejora de las pautas de vida. Los organizadores del censo de 1950, con toda probabilidad imbuidos de la ideología desarrollista del período, anticiparon este proceso en Brasil y colocaron, desde luego, a la mujer en su lugar debido.

En la segunda fase del trabajo se pretende verificar un supuesto esencial para una nueva orientación: la relación entre características individuales y familiares y el modo de producción en que están insertas las mujeres.

Antes de enumerar las características seleccionadas, es preciso recordar que al participar en la producción social las mujeres involucradas en la producción doméstica, se redefine el concepto de población económicamente activa. Se hace caso omiso de que la división que se procura mostrar es convencional, que excluye a las personas que ejecutan trabajo do-

${ }^{26}$ Aarón Cicourel, Method and Measurement in Sociology, Nueva York, Free Press, 1964. 
mástico no remunerado. "Toda la población económicamente activa, por definición, está inserta en la producción social."

Por otro lado, al incluir la producción estatal, se considera también a los que realizan actividades de control gubernamental, jurídicas, políticas, sanitarias, etc., destinadas a mantener y a reproducir el orden constituido. De esta forma se redefine el propio concepto de producción, que pasa a ser un conjunto de actividades no sólo económicas sino políticas y sociales de las cuales resulta el sustento de población y de su reproducción.

La participación en la producción se analiza de la manera siguiente:

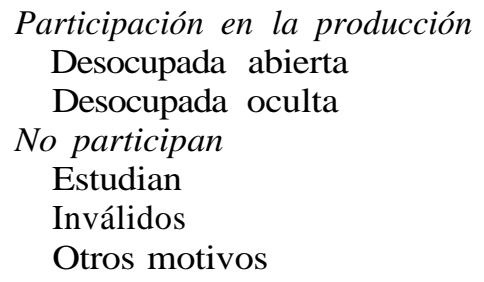

Se procurará caracterizar a las mujeres en los cuatro modos de producción según los atributos individuales siguientes:

Estado civil: casadas-viven con un hombre

solteras - no viven con un hombre

Tipo de ocupación

Ingreso individual

Educación

Edad

Se recurre a características familiares como variables en las cuales las mujeres son consideradas como miembros de una familia y no como individuos. Se construye este tipo de variable sólo para las mujeres casadas. Las solteras son analizadas como individuos.

\section{Hijos}

Las variables relativas a los hijos se construyeron teniendo en cuenta las formas en que éstos pueden influir en el modo de incorporación de la mujer a la producción:

I. Edad de los hijos

ii. Número de hijos

iii. Combinación de las dos anteriores con una noción sobre la etapa del ciclo vital en que se encuentra la familia.

1) sólo con hijos menores de seis años; 
2) con hijos menores de seis años y de 6 a 13 pero no de 14 a 17;

3) con hijos menores de seis años e hijas de 6 a 13 pero sin hijos de 14 a 17

4) con hijos menores de seis años y de 14 a 17 pero no de 6 a 13;

5) con hijos menores de seis años e hijas de 14 a 17 pero sin hijos de 6 a 13 ;

6) con hijos menores de seis años y de 6 a 13 e hijas de 14 a 17;

7) con hijos menores de 6 años, hijas de 6 a 13 e hijos sólo de 14 a 17

8) con hijos menores de seis años, hijos sólo de 6 a 13 e hijas de 14 a 17

9) con hijos de 6 a 13 años solamente;

10) con hijas de 6 a 13 años solamente;

11) con hijos de 6 a 13 años y de 14 a 17;

12) con hijas de 6 a 13 e hijos de 14 a 17;

13) con hijas de 14 a 17 solamente;

14) con hijos menores de 18 años

\section{iv. Hijas que trabajan}

1) Tiene hijos que trabajan y niños menores de seis años;

2) Tiene hijos que trabajan y no posee niños menores de seis años;

3) Tiene hijas que trabajan y niños menores de seis años;

4) Tiene hijas (de 14 o más años) que no trabajan e hijos menores de seis años.

Ayuda doméstica

1) Tiene una empleada doméstica

2) Tiene dos empleadas domésticas

3) Tiene agregados que realizan trabajo doméstico

4) Tiene otros parientes que realizan trabajo doméstico

Personas ocupadas en el domicilio

1) El jefe no trabaja y no cuenta con ayuda de otra persona;

2) El jefe no trabaja y cuenta con ayuda de otra persona;

3) El jefe no trabaja y cuenta con ayuda de dos personas;

4) El jefe no trabaja y cuenta con la ayuda de más de dos personas;

5) El jefe trabaja y no cuenta con ayuda de otra persona;

6) El jefe trabaja y cuenta con la ayuda de una persona;

7) El jefe trabaja y cuenta con la ayuda de dos personas;

8) El jefe trabaja y cuenta con la ayuda de dos o más personas.

La hipótesis central en esta parte es el supuesto de una relación estrecha entre la banda de ingresos en que se encuentra la familia, el ciclo vital y 
los modos de producción en que están insertos los diferentes miembros que componen la familia.

Además de las informaciones obtenidas en el inciso anterior, se utilizan de manera fundamental los cruzamientos siguientes:

Participación en la producción por estructura familiar y nivel de ingreso;

Participación relativa del ingreso familiar por estructura familiar y nivel de ingreso. 